\title{
Postanesthetic torsade de pointes in a patient with unrecognized long QT syndrome
} -A case report-

\author{
Jae-Young Lee, Ju-Hyun Lee, Eun-Hye An, Jun-Gol Song, and Pyung Hwan Park \\ Department of Anesthesiology and Pain Medicine, University of Ulsan College of Medicine, Asan Medical Center, Seoul, Korea
}

Torsade de pointes (TdP) is a devastating form of polymorphic ventricular arrhythmia associated with corrected QT (QTc) interval prolongation. TdP usually terminates spontaneously but frequently recurs and may degenerate to ventricular fibrillation. The present report describes a case of $\mathrm{TdP}$ in a patient being transferred to the postanesthetic care unit following an emergency laparoscopic appendectomy. The patient had undergone open heart surgery 1 week before. Retrospective electrocardiogram analysis revealed the patient had QTc and Tpeak-Tend interval prolongation that had gone unrecognized. We believe TdP may have been induced by accentuation of sympathetic nervous system during emergence from general anesthesia. (Korean J Anesthesiol 2011; 60: 294-297)

Key Words: Long QT syndrome, Torsade de pointes, Tpeak-Tend interval, Ventricular fibrillation.

Torsade de pointes (TdP) is a unique type of polymorphic ventricular tachycardia observed in patients with a prolonged corrected QT (QTc) interval. TdP episodes are usually selfterminating and produce a syncopal episode or pseudoseizure secondary to an abrupt decrease in cerebral blood flow.

The majority of sudden death in long QT syndrome (LQTS) patient results from ventricular fibrillation triggered by TdP. LQTS associated with TdP presents as prolongation of the QTc interval on the electrocardiogram (ECG), and indicates abnormal prolongation of ventricular repolarization due to altered ion flow during the repolarization stage. LQTS can result from mutation of the gene that encodes the ion channel, administration of certain pharmaceuticals (e.g., antiarrhythmic agents, antifungals and antihistamines), electrolyte disorders (e.g., hypokalemia, hypomagnesemia and hypocalcemia) and conditions such as myocardial ischemia. Moreover, it has been reported that anesthetic procedures can cause TdP in LQTS patients due to the anesthetic drug and circumstances that influence the QTc interval change [1-3].

The present report describes a case of TdP in a patient being transferred to the postanesthetic care unit (PACU) following an emergency laparoscopic appendectomy. The patient had undergone open heart surgery a week before. The patient survived, and a retrospective analysis of the heart surgery ECG data showed that he had QTc interval prolongation that had not been identified at the time. We believe the TdP may have

Received: September 9, 2010. Revised: November 12, 2010. Accepted: November 12, 2010.

Corresponding author: Jun-Gol Song, M.D., Department of Anesthesiology and Pain Medicine, University of Ulsan College of Medicine, Asan Medical Center, 388-1, Pungnap-2dong, Songpa-gu, Seoul 138-736, Korea. Tel: 82-2-3010-3869, Fax: 82-2-470-1363, E-mail: jaljary@naver.com (c) This is an open-access article distributed under the terms of the Creative Commons Attribution Non-Commercial License (http:// creativecommons.org/licenses/by-nc/3.0/), which permits unrestricted non-commercial use, distribution, and reproduction in any medium, provided the original work is properly cited. 
been induced by accentuation of sympathetic nervous system (SNS) during emergence from general anesthesia following the appendectomy.

\section{Case Report}

A 53-year-old male (height $169.4 \mathrm{~cm}$, weight $68.2 \mathrm{~kg}$ ) was scheduled for an emergency laparoscopic appendectomy due to acute appendicitis. A week before, he had undergone a mitral valvuloplasty and Maze operation for severe mitral regurgitation and atrial fibrillation. The patient had a history of hypertension and bronchial asthma. A follow-up ECG after the open heart surgery showed a sinus rhythm with a normal QTc interval (Fig. 1A). Postoperative follow-up echocardiography showed mild mitral regurgitation and an ejection fraction of $49 \%$. Laboratory studies returned normal findings except for a prothrombin time of 2.20 international normalized ratio (INR) due to warfarin therapy. Electrolytes including potassium (4.2 $\mathrm{mmol} / \mathrm{L})$, magnesium (1.9 mg/dl) and ionized calcium (0.95 $\mathrm{mmol} / \mathrm{L}$ ) were within normal limits. No preoperative antibiotics were given.

For the emergency appendectomy, the patient was not premedicated. Monitoring included non-invasive blood pressure determination, ECG (lead II), pulse oximetry, and end-tidal carbon dioxide concentration $\left(\mathrm{E}_{\mathrm{T}} \mathrm{CO}_{2}\right)$ measurement. The vital signs included a blood pressure (BP) of 125/75 mmHg, a regular sinus rhythm heart rate (HR) of 85 beats/min, and peripheral oxygen saturation $\left(\mathrm{SpO}_{2}\right)$ of $98 \%$. Anesthesia was induced using mask administration of $100 \%$ oxygen. Etomidate $(12 \mathrm{mg})$ and rocuronium (40 mg) were administered intravenously, and the trachea was intubated using an $8.0 \mathrm{~mm}$ cuffed endotracheal tube.

Anesthesia was maintained using $1 \mathrm{~L} / \mathrm{min}_{2}, 1 \mathrm{~L} / \mathrm{min}_{2} \mathrm{O}$, and $1.5-2.0$ vol\% sevoflurane. Ventilation was mechanically performed at a tidal volume of $600 \mathrm{ml}$ and a rate of 12 breath/ min. The $\mathrm{E}_{\mathrm{T}} \mathrm{CO}_{2}$ was maintained between 31-35 mmHg. At the commencement of surgery, the patient had a BP of 100/65 $\mathrm{mmHg}$, a regular sinus rhythm HR of 95 beats/min and $100 \%$ $\mathrm{SpO}_{2}$. The operation took approximately 1 hour, and proceeded unremarkably. The neuromuscular blocker was then reversed using $15 \mathrm{mg}$ pyridostigmine and $0.4 \mathrm{mg}$ glycopyrrolate. Extubation was performed without complications. At that time, the patient had a $\mathrm{BP}$ of $140 / 100 \mathrm{mmHg}, 100 \% \mathrm{SpO}_{2}$, and the ECG showed sinus tachycardia with a HR of 160 beats/min. As the patient recovered from anesthesia completely including alert mentation, spontaneous breathing, reversal of muscle tone, he was transferred to the PACU.

While applying the monitoring instrument immediately after entering the PACU, the patient lost consciousness, ceased spontaneous breathing and a carotid pulse could not be palpated. BP was unmeasurable, the $\mathrm{SpO}_{2}$ was $36 \%$ and the ECG showed ventricular fibrillation. Mask ventilation was promptly initiated using an ambu-bag, chest compression was performed, and a direct current of $360 \mathrm{~J}$ was applied 3 times using a monophasic defibrillator. The ECG showed resolution of the ventricular fibrillation and appearance of a sinus rhythm. However, shortly thereafter TdP-type polymorphic ventricular tachycardia developed (Fig. 1B). Defibrillation was performed 3 times, and $1 \mathrm{mg}$ epinephrine and $110 \mathrm{mg}$ lidocaine were intravenously administered. Additionally, magnesium sulfate $2 \mathrm{~g}$ was given intravenously over $10 \mathrm{~min}$. The sinus rhythm returned with a HR of 80 beats/min, the BP increased to $166 / 80 \mathrm{mmHg}$ and the $\mathrm{SpO}_{2}$ to $95 \%$. The patient was moved to the intensive care unit (ICU) with endotracheal intubation in a stable condition. The BP was 110/67 mmHg, the $\mathrm{SpO}_{2}$ was $98 \%$, and an ECG showed QTc interval prolongation (617 ms) and a HR of 111 beats/ min on arrival at the ICU. Norepinephrine $(0.04 \mu \mathrm{g} / \mathrm{kg} / \mathrm{min})$, isosorbide dinitrate ( $1 \mu \mathrm{g} / \mathrm{kg} / \mathrm{min})$ and magnesium sulfate $(2 \mathrm{~g})$ were administered intravenously. The vital signs stabilized and norepinephrine infusion was ceased at 3 hours after ICU arrival.

A

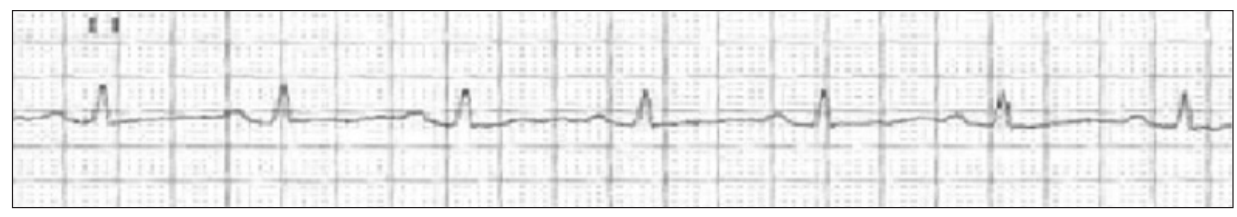

B

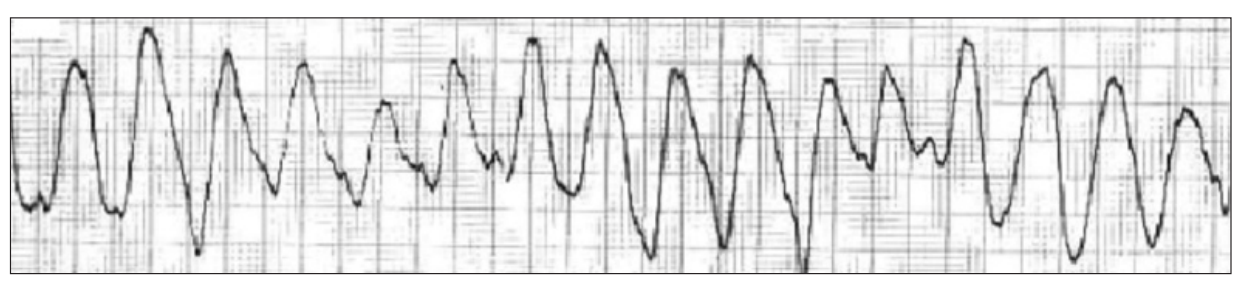

Fig. 1. Changes in electrocardiography (ECG) during the perioperative period. (A) Preoperative ECG (lead II) rhythm strip demonstrated a normal QT interval prolongation. (B) Postoperative ECG monitoring (lead II) demonstrated torsade de pointes in the postanesthetic care unit. 


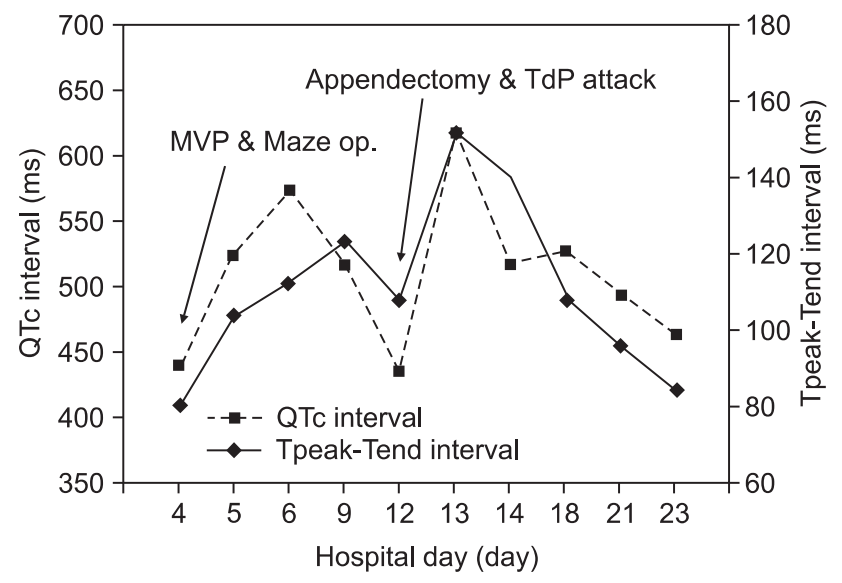

Fig. 2. Serial changes in QTc interval and Tpeak-Tend interval. The Tpeak-Tend interval is the longest interval between peak point of $\mathrm{T}$ wave and end point of T wave of ECG. Note that the Tpeak-Tend and QTc interval were prolonged postoperatively. QTc: corrected QT, MVP: mitral valvuloplasty, Maze op.: Maze operation, TdP: torsade de pointes.

There was no necessity for supplement of electrolytes during ICU care, because all electrolytes were within normal limits including potassium $(4.1 \mathrm{mmol} / \mathrm{L})$, magnesium $(2.2 \mathrm{mg} / \mathrm{dl})$ and ionized calcium $(0.9 \mathrm{mmol} / \mathrm{L})$. In addition, no other drugs known to prolong QTc interval were administered. The patient was moved to the general ward on the 3rd postoperative day without relapse of TdP.

We retrospectively investigated the QTc interval and TpeakTend interval (Tpe interval) identified on the ECG data (Fig. 2). The ECG showed QTc interval prolongation after the open heart surgery, although it had normalized by the day of the emergency appendectomy. The patient was discharged without relapse of TdP.

\section{Discussion}

LQTS is an arrhythmogenic cardiovascular disorder with prolongation of ventricular repolarization, including a QTc interval prolongation longer than $440 \mathrm{~ms}$, and an abnormal $\mathrm{T}$ wave (broad, notched, small) that causes ventricular tachycardia such as TdP and ventricular fibrillation resulting in syncope or sudden death [4]. LQTS has traditionally been categorized as either congenital or acquired. Congenital LQTS is the result of gene mutations that cause cardiac ion channel dysfunction. Acquired LQTS is caused by the administration of certain drugs (e.g., antiarrhythmic agents, antibiotics and tricyclic antidepressants), electrolyte disorders (e.g., hypocalcemia, hypomagnesemia and hypokalemia), and myocardial ischemia. However, it has recently been suggested that acquired LQTS is linked to a silent mutation in the gene that causes congenital LQTS [5]. LQTS can also be divided into adrenergic- and pause-dependent types. Congenital LQTS such as Jervell-Lange-Nielsen syndrome and Romano-Ward syndrome belong to the adrenergic-dependent type that causes QTc interval prolongation and ventricular arrhythmia through stimulation of the adrenergic receptor. Most acquired LQTS is the pause-dependent type that causes QTc interval prolongation and arrhythmia via bradyarrhythmia or sinus pause.

Transmural dispersion (i.e., significant differences in repolarization in the various layers of the myocardium) is a feature of TdP and ventricular fibrillation in patients with LQTS [6,7]. A decrease in the outward potassium ion current or an increase in the slow calcium ion inward current during repolarization resulting from mutation of the gene encoding the ion channel prolongs the action potential duration that leads to early afterdepolarization (EAD). In addition to the reentry mechanism, EAD and transmural dispersion induce TdP [8]. Transmural dispersion is indicated by the prolonged Tpe interval on ECG. Therefore, the Tpe interval in patients with LQTS is a predictive factor for ventricular arrhythmia [9]. In healthy individuals, the reference values for the Tpe interval are $94 \pm 10 \mathrm{~ms}$ in men and $92 \pm 11 \mathrm{~ms}$ in women [10]. In the present patient, the Tpe interval was prolonged (mean: $110.8 \mathrm{~ms}$, range: 80-152 ms), as expected.

Therefore, we undertook a retrospective analysis of the ECG data and found there was continued QTc interval prolongation after the Maze operation. The mechanisms underlying QTc interval prolongation after conversion to a sinus rhythm are not clearly understood. The present patient showed no evidence of congenital or acquired disorders according to family history, personal history or preoperative examination. A previous report showed that post-aortic valve replacement bradycardia (atrial fibrillation with slow ventricular response) induced TdP due to trauma or ischemia of conduction pathway [11]. However, ECG of the present patient was normal except QTc interval prolongation after open heart surgery. Given that no issues arose during induction, maintenance and emergence of anesthesia (e.g., hypoxia, hypotension, ECG changes of cardiac ischemia, arrhythmia), and that HR increased to 160 beats/min just before TdP occurrence, we assume that TdP was the result of excessive accentuation of the SNS in a patient who already had QTc interval prolongation. Although postoperative pain can also be one of the causes of activation of the SNS, we assumed that glycopyrrolate has a main causal relationship with accentuating the SNS, because HR increased abruptly immediate after administration of glycopyrrolate. Glycopyrrolate has been shown to lengthen the QT interval in healthy subjects [12]. Moreover, previous reports have shown that TdP occurred in a patient with QTc interval prolongation as a result of accentuating the SNS by atropine induced ventricular tachycardia [12]. Taken together, impairment of the 
parasympathetic control of the HR caused by anticholinergics might influence the cardiac sympathetic tone and hence the QTc interval, therefore, anticholinergic should be used with caution to prevent any extreme changes in HR.

Anesthetic management of adrenergic-dependent LQTS patients requires avoidance of drugs that affect QTc interval prolongation. In addition, more than two types of ECG leads, an invasive blood pressure monitoring device, a central venous catheter and a cardiac defibrillator should be prepared. $\beta$-blockers are the drugs of choice to prevent TdP through blocking stimulation of the SNS. Preoperative anxiety in a patient also activates the SNS, hence pretreatment with an anxiolytic agent such as midazolam is recommended. All inhalation anesthetics should be used with caution in LQTS patients because they are known to prolong the QTc interval by reducing the potassium ion current or increasing transmural dispersion during repolarization, though the degree of effect varies. Chae et al. reported that sevoflurane prolonged the action potential duration and QTc interval on ECG by suppressing the transient outward potassium current at phase 1 and early phase 2 of repolarization in mouse ventricular myocytes [13]. Atropine and glycopyrrolate are also known to prolong the QTc interval in LQTS patients by accentuating the SNS.

Magnesium sulfate is the treatment of choice for TdP and lidocaine can also be used. Serum potassium should be checked and maintained as a high normal level [14]. Temporary transvenous pacing is an effective way of controlling TdP. Pacing is particularly effective in pause-dependent LQTS [15]. If all of these treatments are ineffective, defibrillator should be carried out.

In conclusion, various anesthetic drugs and conditions during the perioperative period can prolong the QTc interval and activate the SNS. This leads to an increased risk of fatal consequences such as TdP and ventricular fibrillation in patients with LQTS. Therefore, measures should be undertaken to ensure LQTS does not go unrecognized.

\section{References}

1. Gil NS, Oh AY, Kim HS, Kim CS. Cardiac arrest under anesthesia in a child with previously undiagnosed long QT syndrome. Korean J
Anesthesiol 2005; 49: 274-7.

2. Kleinsasser A, Kuenszberg E, Loeckinger A, Keller C, Hoermann C, Lindner KH, et al. Sevoflurane, but not propofol, significantly prolongs the Q-T interval. Anesth Analg 2000; 90: 25-7.

3. Katz RI, Quijano I, Barcelon N, Biancaniello T. Ventricular tachycardia during general anesthesia in a patient with congenital long QT syndrome. Can J Anaesth 2003; 50: 398-403.

4. Garson A Jr, Dick M II, Fournier A, Gillette PC, Hamilton R, Kugler JD, et al. The long QT syndrome in children. An international study of 287 patients. Circulation 1993; 87: 1866-72.

5. Chevalier P, Rodriguez C, Bontemps L, Miquel M, Kirkorian G, Rousson R, et al. Non-invasive testing of acquired long QT syndrome: evidence for multiple arrhythmogenic substrates. Cardiovasc Res 2001; 50: 386-98.

6. Akar FG, Yan GX, Antzelevitch C, Rosenbaum DS. Unique topographical distribution of $\mathrm{M}$ cells underlies reentrant mechanism of torsade de pointes in the long-QT syndrome. Circulation 2002; 105 : 1247-53.

7. Antzelevitch C, Shimizu W. Cellular mechanisms underlying the long QT syndrome. Curr Opin Cardiol 2002; 17: 43-51.

8. Viswanathan PC, Ruby Y. Cellular arrhythmogenic effects of congenital and acquired long-QT syndrome in the heterogeneous myocardium. Circulation 2000; 101: 1192-8.

9. Yamaguchi M, Shimizu M, Ino H, Terai H, Uchiyama K, Oe K, et al. T wave peak-to-end interval and QT dispersion in acquired long QT syndrome: a new index for arrhythmogenicity. Clin Sci (Lond) 2003; 105: 671-6.

10. Haarmark C, Graff C, Andersen MP, Hardahl T, Struijk JJ, Toft E, et al. Reference values of electrocardiogram repolarization variables in a healthy population. J Electrocardiol 2010; 43: 31-9.

11. Amchentsev A, Haq SA, Holt JJ, Khan A, Gelles J, Kassotis J. Postaortic valve replacement bradycardia induced torsades de pointes. Angiology 2008; 59: 111-3

12. Saarnivaara L, Simola M. Effects of four anticholinesteraseanticholinergic combinations and tracheal extubation on QTc interval of the ECG, heart rate and arterial pressure. Acta Anaesthesiol Scand 1998; 42: 460-3.

13. Chae JE, Kim CH, Min KT, Park WK. Electrophysiologic mechanism of sevoflurane on prolongation of the QT interval: $\mathrm{K}^{+}$currents in rat ventricular myocytes. Korean J Anesthesiol 2006; 50: 454-62.

14. Hasegawa J, Takami T, Kaneda T, Yamane W, Hoshio A, Igawa O, et al. Treatment of torsade de pointes with intravenous magnesium in idiopathic long QT syndrome. Jpn Circ J 1991; 55: 1057-60.

15. Koo BN, Kang OC, Kim KB, Park WK, Kil HK. Anesthetic management of a patient with congenital long QT syndrome. Korean J Anesthesiol 2004; 46: 743-6. 